\title{
Symmetry Conserving Dynamical Mappings
}

\author{
Zoheir Aouissat ${ }^{a}$ \\ a Institut für Kernphysik, Technische Universität Darmstadt, Schloßgartenstraße 9, \\ D-64289 Darmstadt, Germany
}

Using the concept of dynamical mappings, two symmetry conserving nonperturbative approaches are presented. The first is based on the $1 / N$-expansion and sorted out using Holstein-Primakoff mapping. The second consists of dynamically mapping the canonical fields into the corresponding currents. It is argued, either by comparing the Fock spaces or the observables, that the latter constitutes a higher approach which transcends the $1 / N$ expansion and contains the dynamics generated by the Gaussian functional approach.

\section{INTRODUCTION}

Solving a theory for interacting fields is one of the major challenges in quantum field theory (QFT). It is in fact amazing to realize that, besides the coupling constant perturbation (CCP), the analytical (approximate) solutions at our disposal are of a semi-classical nature. These are based on expansions in the number of colors $\left(N_{c}\right)$, flavors $\left(N_{f}\right)$, or simply charges $(N)$, depending on the problem at hand. I will refer to these generically by the $1 / N$-expansion. The symmetries are known to be preserved by these approaches since the dynamics, like in the case of the CCP, is sorted out according to an expansion in an arbitrary parameter. It is well known that the usual CCP is supported by a Fock space made of an "uncorrelated" vacuum $|0\rangle$ and excited states $|\nu\rangle$ build by the action of creation operators of the quantized canonical fields. Going to higer order in the loops one builds correlations perturbatively which leads to a gradual redefinition of the vaccum at each order. An interesting question to raise is: can one envisage a similar construction for the Fock space in the case of a nonperturbative approach e.g. the semi-classical ones? In the following I will explore such a possibility and show that the idea is fertile and can even help guessing a promising "new" nonperturbative approach. The latter, in contrast to the semi-classical one, is an approximate solution with a full quantum character. It transcends the $1 / N$-expansion and contains the Gaussian functional approach (GFA). In fact, it was the need for such kind of solutions of QFT which triggered the interest into the GFA. Unfortunately, it was quickly realized that the latter, being order mixing, is not in general able to treat the symmetries correctly [1]. Here I would like to argue that the second nonperturbative symmetry conserving approximation, next to the $1 / N$-expansion, needs in fact much more vaccum-correlations than what the GFA offers. We will see that these correlations are of a RPA-type, selected carefully by dynamically mapping the canonical fields into the currents with the corresponding quantum numbers. The idea of substituting the currents for the canonical fields is in fact not new. It was used in the 
late sixties by Callan, Dashen, Sharp, Sommerfield, and Sugawara [2] in an attempt to build a QFT with currents as dynamical variables giving up the concept of describing the fields with canonical variables. In the following, I would like to draw a slightly different picture. Although I will substitute for the asymptotic fields the corresponding currents, I will not renounce the use of the canonical fields as building blocks of QFT. Mapping these canonical fields into the currents will then help in gathering the dynamics which dress the asymptotic fields while preserving the symmetry. In section 3 , we will see how this can be put to work in building a nonperturbative pion by mapping its canonical field into the axial current. However, I will first, in section 2, revisit the semi-classical $1 / N$-expansion approach using the very concept of dynamical mappings.

As an example, consider the toy model of QFT, the $\Phi^{4}$-theory with a continuous $O(N+1)$ symmetry. The lagrangian density with the appropriate scaling reads

$$
\mathcal{L}=\frac{1}{2}\left[\left(\partial_{\mu} \vec{\pi}\right)^{2}+\left(\partial_{\mu} \sigma\right)^{2}\right]-\frac{\mu^{2}}{2}\left[\vec{\pi}^{2}+\sigma^{2}\right]-\frac{\lambda}{4 N}\left[\vec{\pi}^{2}+\sigma^{2}\right]^{2}+\sqrt{N} c \sigma
$$

where $\vec{\pi}(x)$ stands for a $N$-components pion field and $\sigma(x)$ its chiral partner.

\section{HOLSTEIN-PRIMAKOFF MAPPING}

First let us see how the concept of symmetry conserving dynamical mapping (SCDM) can be used to retrieve the well known $1 / N$-expansion. It is clear that, due to the Bose statistics, the pion wave function induces direct and exchange contributions (Hartree and Fock terms) which are of two distinct orders in the $1 / N$ counting. Therefore to hinder any order mixing in the $1 / N$-expansion one should, whatever the procedure used, only allow the Hartree terms as leading contributions and relegate the Fock terms to the sub-leading orders. This is, however, only possible if the pion wave-function is severely truncated, leading to a particle which doesn't fully enjoy the quantum statistics or, in other words, to a Hartree particle. Sorting out the dynamics according to this scheme can be achieved by means of a pion-pair bosonization via the so-called Holstein-Primakoff mapping (HPM). The latter appeared first in the early fourties [3] as a realization of the $S U(2)$ algebra for quasi-spins. It was forgotten ever since and reappeared in the sixties in the nuclear many-body problem where it was used for bosonizing fermion-pairs (see [4] for a review). In the present case the HPM for pion-pairs reads (see [5] for details):

$$
\vec{a}_{q}^{+} \vec{a}_{p}^{+} \rightarrow\left(A^{+} \sqrt{N+A^{+} A}\right)_{q, p}, \quad \vec{a}_{q} \vec{a}_{p} \rightarrow\left(\vec{a}_{p}^{+} \vec{a}_{q}^{+}\right)^{+}, \quad \vec{a}_{q}^{+} \vec{a}_{p} \rightarrow\left(A^{+} A\right)_{q, p},
$$

where $\vec{a}_{q}^{+}, \vec{a}_{q}$ stand for the pion creation and annihilation operators, while $A_{q, p}$ and $A_{q, p}^{+}$are real boson operators obeying the Heisenberg-Weyl algebra. This mapping is made in such a way that the original algebra, obeyed by the pairs of operators at the 1.h.s of eq.(2), is also realized by the ansatz at the r.h.s. The square root is to be understood as a formal power series in the operators. Thus the Hamiltonian of the vector model derived from eq.(1) will naturally inherit a formal expansion of the form: $H=\mathcal{H}^{(0)}+\mathcal{H}^{(1)}+\mathcal{H}^{(2)}+\mathcal{H}^{(3)}+\mathcal{H}^{(4)}+.$. , where the superscripts indicate the powers of the operators $A$ and $A^{+}$and also $b$ and $b^{+}$ for the sigma field. At this stage, the content in $N$ of each order $\mathcal{H}^{(p)}$ is not yet specified. Also the formal expansion is in reality not unique since the operators are not in normal order. Therefore a definition of a vacuum is mandatory if one wishes to make any use of 
the above expansion. By defining a vacuum for the $A$ and $b$ operators one makes the HPM dynamical. To meet the desired $1 / N$-expansion approach this step is indeed decisive. In other words not any vacuum and thus not any Fock space is able to support this approach. It was shown in [5] that the vacuum of the $1 / N$-expansion is a coherent state

$$
\left.\left|\psi>=\exp \left[\sum_{q} d_{\pi \pi}(q) A_{q,-q}^{+}+\left\langle b_{0}\right\rangle b_{0}^{+}\right]\right| 0\right\rangle,
$$

which can accommodate condensates of the sigma field, denoted here by $\left\langle b_{0}\right\rangle$, as well as pairs of Hartree pions, denoted by $d_{\pi \pi}(q)$. Assuming this, the Hamiltonian displays then a parallel (and unambiguous) expansion in the powers of $N$, such that

$$
H=N H^{(0)}+\sqrt{N} H^{(1)}+H^{(2)}+\frac{1}{\sqrt{N}} H^{(3)}+\frac{1}{N} H^{(4)}+\ldots
$$

Here the terms $H^{(p)}$ have no content in $N$. To gather the dynamics, one has to diagonalise $H$. Odd powers in $\sqrt{N}$ are completely off-diagonal and therefore ought to disappear. The leading order dynamics is contained in the three lowest terms, thus we disregard here all higher terms. The term $H^{(1)}$ is washed out by performing a variational Hartree-Bogoliubov (HB) calculation, using $\left\langle b_{0}\right\rangle$ and $d_{\pi \pi}(q)$ as variational parameters in minimizing the ground state energy. The bilinear $H^{(2)}$ is diagonalized by applying a canonical Bogoliubov rotation [5] which mixes the operators $b, b^{+}$and $A, A^{+}$such that:

$$
Q_{\vec{p}}^{+}=X_{\vec{p}} b_{\vec{p}}^{+}-Y_{\vec{p}} b_{-\vec{p}}+\sum_{q}\left[U_{\vec{q}, \vec{p}} A_{\vec{q}, \vec{p}-\vec{q}}^{+}-V_{\vec{q}, \vec{p}} A_{-\vec{q},-\vec{p}+\vec{q}}\right]
$$

This is nothing but a $\pi \pi$ RPA-scattering equation coupled to a Dyson equation for the sigma mode. The vacuum of the theory is accordingly modified. The latter, denoted by $|R P A\rangle$, is implicitly defined by $Q_{\vec{p}}|R P A\rangle=0$ and explicitly obtained via a unitary transformation of the coherent state: $|R P A\rangle=U_{\text {unitary }}|\psi\rangle$.

This exhausts the leading order dynamics. In a cutoff theory, the $|R P A\rangle$-vacuum, obtained so far, possesses a broken phase with a finite sigma-condensate $(\langle\sigma\rangle \neq 0)$ and two curvatures; one is the Goldstone boson mass, obtained in the HB mean-field, and the second is the sigma mass, obtained in the RPA. These are given by

$$
\begin{aligned}
& m_{\pi}^{2}=\mu^{2}+\lambda\left[I_{\pi}+\langle\sigma\rangle^{2}\right], \quad \frac{c}{\langle\sigma\rangle}=\mu^{2}+\lambda\left[I_{\pi}+\langle\sigma\rangle^{2}\right] \\
& m_{\sigma}^{2}=\mu^{2}+\lambda\left[I_{\pi}+3\langle\sigma\rangle^{2}\right]+\frac{2 \lambda^{4}\langle\sigma\rangle^{2} \Sigma_{\pi \pi}\left(m_{\sigma}^{2}\right)}{1-\lambda^{2} \Sigma_{\pi \pi}\left(m_{\sigma}^{2}\right)}
\end{aligned}
$$

Here $I_{\pi}$ is the tadpole of the HB-pion and $\Sigma_{\pi \pi}\left(p^{2}\right)$ stands for the convoluted two HBpion propagator (RPA bubble of HB-pions). Naturally, this approach preserves the whole hierarchy of Ward identities. In particular, the lowest one which expresses the current conservation (in PCAC sense), $D_{\pi}^{-1}(0)=\frac{c}{\langle\sigma\rangle}$, holds. Figures 1.a and 1.b show the summed class of diagrams.

\footnotetext{
1 This transformations is constructed as a product of three unitary inequivalent transformations. The first is a unitary squeezing transformation in the $\left(b, b^{+}\right)$-sector, the second is a similar one in the $\left(A, A^{+}\right)$-sector and the third is a unitary transformation which mixes both sectors [6].
} 
Fig. 1.a. BCS solution in a Hartree-Bogoliubov (HB) approximation. The pion mass and the condensate are given by two coupled selfconsistent equations. There is no dynamical mass generation therefore the pion is a Goldstone mode

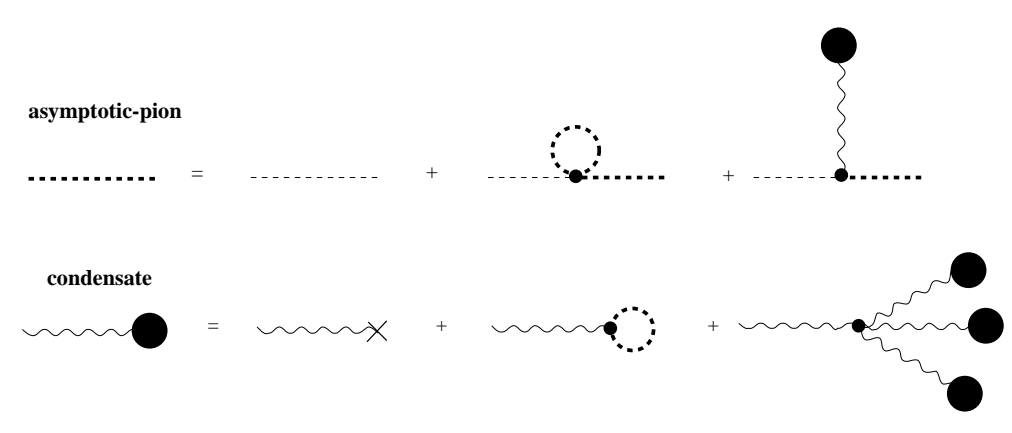

Fig. 1.b. Dyson equation for the $\sigma$ mode coupled to a RPA equation for $\pi \pi$ scattering. According to this scheme, the sigma mass is build perturbatively (in contrast to the self-consistent building of the pion and the condensate in Figure 1.a.)

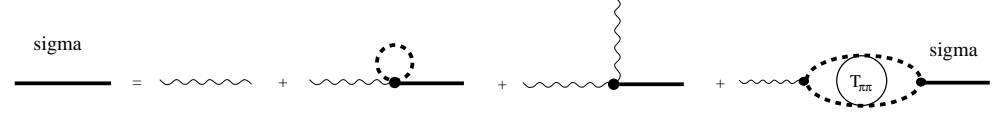

\section{PIONIC-QRPA MAPPING}

The approach presented in the previous section is very appealing from many aspects and particularly from its symmetry conserving character. However, it has a serious drawback. The pion, constructed so far, is a Hartree particle thus a semi-quantum (semi-classical) "object". It is also the building block for all higher n-point functions, as suggested by the whole hierarchy of Ward identities. Attempts made with the GFA failed so far to correct for this [1]. Indeed, assuming the full wave function of the pion (instead of truncating it) induces an uncontrollable order mixing which inevitably "destroys the symmetry". As stated in the introduction, one way out is to map the canonical pion field into the axial current. This idea is supported by the exact (Goldstone) statement: $Q_{5}^{a}|v a c\rangle \propto\left|\pi^{a}\right\rangle$, which allows to build a pion state by acting with the symmetry generator on the full correlated vacuum of the theory. In an effective model, the generator $Q_{5}^{a}$ is simply given by Noether's theorem. Therefore one can use the field structure of $Q_{5}^{a}$ to model an excitation operator for the asymptotic pion field. In the present case, the creation operator of the iso-vector pion takes the form

$$
\vec{Q}_{\pi}^{+}=X_{\pi}^{(1)} \vec{a}_{0}^{+}-Y_{\pi}^{(1)} \vec{a}_{0}+\sum_{q}\left[X_{\pi}^{(2)}(q) b_{q}^{+} \vec{a}_{-q}^{+}-Y_{\pi}^{(2)}(q) b_{-q} \vec{a}_{q}+X_{\pi}^{(3)}(q) b_{q}^{+} \vec{a}_{q}-Y_{\pi}^{(3)}(q) b_{q} \vec{a}_{-q}^{+}\right] .
$$

Here the operators $\vec{a}_{q}$ and $b_{q}$ represent the canonical pion- and sigma- fields. The $X$ and $Y$ amplitudes are fixed dynamically. Using these as variational variables to minimize the the pion-state energy $\left(\delta \frac{\langle\pi|H| \pi\rangle}{\langle\pi \mid \pi\rangle}=0\right)$ leads to the so-called Rowe equation of motion

$$
\left\langle R P A\left|\left[\delta \vec{Q}_{\pi},\left[H, \vec{Q}_{\pi}^{+}\right]\right]\right| R P A\right\rangle=m_{\pi}\left\langle R P A\left|\left[\delta \vec{Q}_{\pi}, \vec{Q}_{\pi}^{+}\right]\right| R P A\right\rangle
$$

where $\mathrm{H}$ is the Hamiltonian of the model, $m_{\pi}$ is the pion mass (excitation energy to create a pion at rest) and $|R P A\rangle$ is an approximate ansatz to the full correlated vacuum $|v a c\rangle$, defined implicitly by : $\vec{Q}_{\pi}|R P A\rangle=0$. The eigenvalue problem in eq.(9), in its present variational form, is known as the self-consistent RPA which can not be solved in practice. Therefore one uses in general the quasi-boson assumption which approximates the bilinear 
$\vec{Q}_{\pi}$ by a boson. Thus eq.(9) is linearized. In the exact chiral limit $(c=0)$, one of its solutions, if successfully normalized, has zero energy $\left(m_{\pi}=0\right)$. The normalization of this Goldstone solution can in fact be achieved by optimizing the RPA basis. This is done by dynamically mapping the original canonical pion $\left(\vec{a}, \vec{a}^{+}\right)$and sigma $\left(b, b^{+}\right)$fields into Hartree-Fock-Bogoliubov (HFB) fieldst

$$
\vec{\alpha}_{q}^{+}=u_{q} \vec{a}_{q}^{+}-v_{q} \vec{a}_{-q}, \quad \beta_{q}^{+}=x_{q} b_{q}^{+}-y_{q} b_{-q}-w^{*} \delta_{q 0} .
$$

Here $u, v, x, y, w$ are variational functions chosen to minimize the energy of the vacuum of the theory. The latter is given, up to an unimportant factor, by the squeezed state

$$
|\Phi\rangle=\exp \left[\sum_{q} \frac{v_{q}}{2 u_{q}} \vec{a}_{q}^{+} \vec{a}_{-q}^{+}+\frac{y_{q}}{2 x_{q}} b_{q}^{+} b_{-q}^{+}+\frac{w}{2 x_{0}} b_{0}^{+}\right]|0\rangle .
$$

The dynamics gathered at this HFB mean-field appear in the following set of equations:

$$
\begin{aligned}
\mathcal{E}_{\pi}^{2} & =\mu^{2}+\lambda\left[\frac{N+2}{N} I_{\pi}+\frac{1}{N} I_{\sigma}+\langle\sigma\rangle^{2}\right], \quad \mathcal{E}_{\sigma}^{2}=\mu^{2}+\lambda\left[I_{\pi}+\frac{3}{N} I_{\sigma}+3\langle\sigma\rangle^{2}\right], \\
\frac{c}{\langle\sigma\rangle} & =\mu^{2}+\lambda\left[I_{\pi}+\frac{3}{N} I_{\sigma}+\langle\sigma\rangle^{2}\right] .
\end{aligned}
$$

which consist of three coupled self-consistent BCS gap equations that give the condensate $(\langle\sigma\rangle)$ and the two curvatures $\left(\mathcal{E}_{\pi}, \mathcal{E}_{\sigma}\right)$ of the squeezed vacuum $|\Phi\rangle$.

Here $I_{\pi}$ and $I_{\sigma}$ are, respectively, the tadpoles for the pion and sigma quasi-particles (with the Hartree and Fock terms considered together). This is precisely the dynamics generated by the GFA where $\mathcal{E}_{\pi}$ stands for the asymptotic pion mass. This is, however, clearly wrong. Indeed, in the exact chiral limit $(c=0)$ and for a finite condensate, the curvature $\mathcal{E}_{\pi}$ does not vanish (see also [1]). Therefore the squeezed state and equally the Gaussian functional can not be regarded as a viable vacuum for the theory since the Goldstone theorem is violated. However, the RPA ground state, as implicitly defined by $\vec{Q}_{\pi}|R P A\rangle=0$, is a good candidate for a vacuum with broken symmetry. The latter, in the case of the quasi-boson assumption (used here), is explicitly obtained by an unitary transformation of the squeezed state: $|R P A\rangle=U_{\text {unitary }}|\Phi\rangle$ 3. The curvature along the valley of this ground-state is given by the RPA eigenvalue $m_{\pi}$ and reads:

$$
m_{\pi}^{2}=\frac{c}{\langle\sigma\rangle}+\frac{2 \lambda^{2}}{N} \frac{\left[\mathcal{E}_{\pi}^{2}-\mathcal{E}_{\sigma}^{2}\right]\left[\Sigma_{\pi \sigma}(0)-\Sigma_{\pi \sigma}\left(m_{\pi}^{2}\right)\right]}{1-\frac{2 \lambda^{2}}{N} \Sigma_{\pi \sigma}\left(m_{\pi}^{2}\right)}
$$

where $\Sigma_{\pi \sigma}\left(p^{2}\right)$ is the convoluted quasi-pion and quasi-sigma propagators (see figure 2). It is clear, from eq.(13), that the asymptotic pion is not only highly nonperturbative in the coupling $\lambda$ but also has a non-trivial content in $N$, in contrast to the HB-pion of section 2. It is, however, still a Goldstone mode, since for $c=0$ and $\langle\sigma\rangle \neq 0$ a zero pion mass

${ }^{2}$ This is in fact the minimal procedure to achieve the normalization of the Goldstone solution. Other normalization procedures, based on Higher-RPA, and which allow to gather more dynamics than the present approach, are also possible. Further discussion on this point is deferred to a coming work.

${ }^{3}$ In the case of the QBA assumption, the way of building the unitary transformation is similar to the one sketched in footnote 1. Because of the infinite degrees of freedom, both vacuums are in fact inequivalent. 
exists. Furthermore it is easily verified that the Ward identity, $D_{\pi}^{-1}(0)=\frac{c}{\langle\sigma\rangle}$, holds here too.

Fig.2. Diagrammatic representation of the collected dynamics. In step I, the optimized quasiparticle basis is build as a BCS solution in the HFB-approximation. In step II, the quasiparticle states are scattered in a LippmannSchwinger equation. In step III, a mass operator is build out of the full vertex $T_{\pi \sigma}$ and inserted in a Dyson equation to generate the asymptotic Goldstone pion.

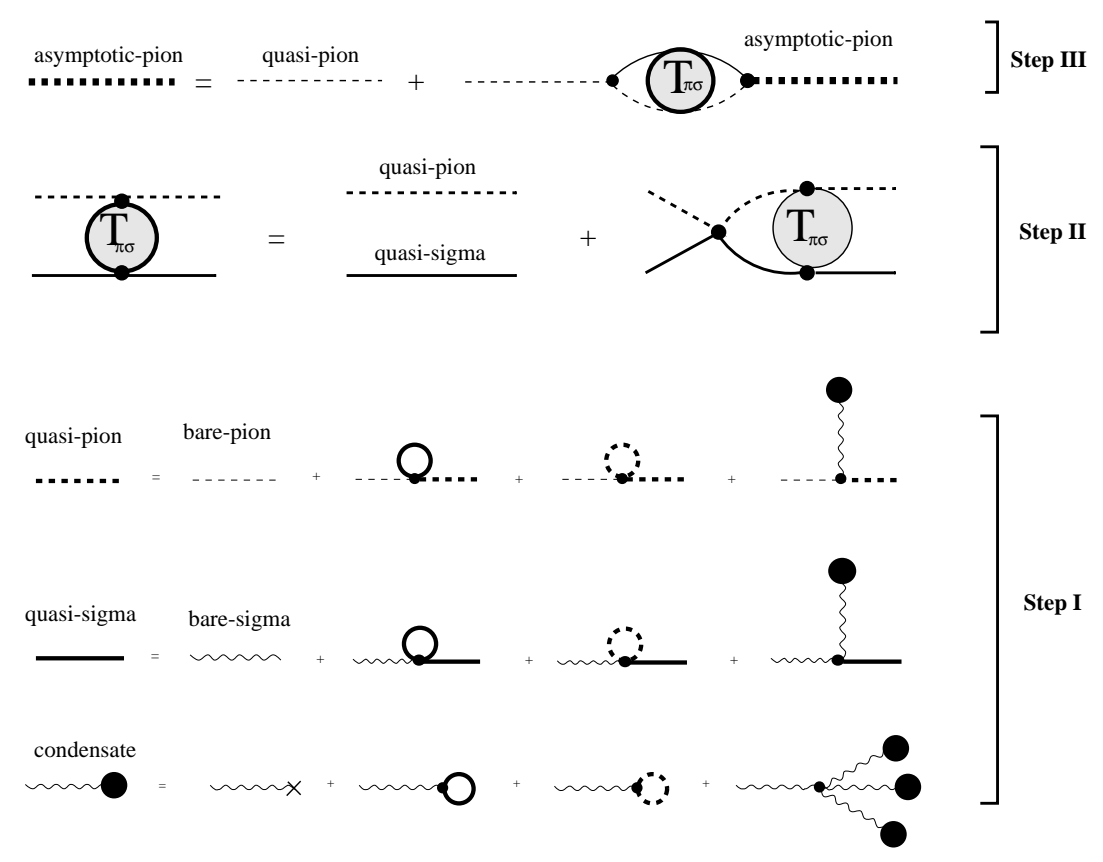

\section{CONCLUSION}

There is obviously an urgent need for developing symmetry conserving nonperturbative approaches with tractable analytical solutions to QFT. I exposed here the concept of SCDM which is a promising tool that gives a helpful insight on the structure of the Fock space. Besides the HPM which leads to the $1 / N$-expansion, I presented a second SCDM that relies on a systematic scheme which consists of mapping the canonical fields into the corresponding currents. The latter mapping was made dynamical in the quasiparticle RPA. The vacuum of the theory was then found to have more correlations than the vacuums of the $1 / N$-expansion and the GFA alike. Extensions to higher RPA, finite temperature and baryon density as well as to richer dynamics are possible [7].

Acknowledgements : I would like to thank G. Chanfray, P. Schuck and J. Wambach for their interest in this work and for their continuous support.

\section{REFERENCES}

1. G. A. Camelia, Phys. Lett. B407 (1997) 268, I. Kogan et al. PRD. 6 (1995) 3719.

2. C. G. Callan, R. F. Dashen, D. H. Sharp, 165 (1968) 1883, H. Sugawara, Phys. Rev. 170 (1968) 1659, C. M. Sommerfield, Phys. Rev. 176 (1968) 2019.

3. T. Holstein, H. Primakoff, Phys. Rev. 58 (1940) 1098.

4. A. Klein, E.R. Marshalek, Rev. Mod. Phys. Vol. 63, No. 2 (1991) 375.

5. Z. Aouissat, P. Schuck, J. Wambach, Nucl. Phys. A618 (1997) .402

6. M. Blasone, P. H. Henning, G. Vitiello, hep-ph/9605335.

7. Z. Aouissat, O. Bohr, J. Wambach, hep-ph/9710419, Z. Aouissat, M. Belkacem, in preparation. 